\title{
Le Financement Des Pme « Africaines » : La Nécessité Du Crédit-Bail Et La Bourse Des Valeurs
}

\author{
Dr. Pony Lucas \\ Enseignant chargé de cours /Expert en Banque et Micro finance, \\ Université de Dschang au Cameroun
}

doi: 10.19044/esj.2016.v12n19p190 URL:http://dx.doi.org/10.19044/esj.2016.v12n19p190

\begin{abstract}
Confronted with the growing scarcity of resources in the global scale, the international political dialogue, in accordance with the Monterry consensus (2002) and the Doha conference (2008), recommends the reinforcement of internal resources mobilization in developing countries to achieve the Millennium Development Goals. In the case of Cameroon, emphasis is put on leasing as a form of financing, so as to enable a company to acquire production equipment; that is, goods that will enhance production in a company. Moreover, the financing of SME centered on the stock exchange speculations as possible alternatives of long-term SME financing, i.e. for more than five years and based on tangible fixed assets, can be an orientation to the fight against poverty in Africa. To succeed, the main objective of this study consist of evaluating the leasing performance between 1994 and 2008 practiced in cameroonian banking system, following the 2007-2008 economic and financial world crisis. What will not be possible without insisting on the Durban Watson test for the period of 1994-2008; thus, evaluating the role of leasing and the Stock Market in the SME financing. The present study identifies suitable strategies to improve the output and the effectiveness of leasing. In addition, investigations will lead to results that can contribute to decrease the bank loan application shares rejected by the banks in short and long periods; the stock market will take over. The methodological approach will focus on data collection, statistical and econometric analysis which estimates the output and the performance of leasing in development, financing and poverty in Cameroon. The recommendations will reinforce the Cameroon banks' capacities to finance development and the fight against poverty by leasing.
\end{abstract}

Keywords: Financing, leasing, Stock Market, Micro-financing, poverty, taxation 


\section{Résumé}

Confronté à la raréfaction des ressources au niveau mondial, le dialogue politique international, conformément au consensus de Monterrey(2002) et la conférence de Doha (2008) préconise le renforcement de la mobilisation des ressources internes dans les pays en développement pour atteindre les objectifs du millénaire pour le développement Dans le cas du Cameroun, un accent est à mettre sur le crédit bail comme une des formes de financement permettant à une entreprise de se doter d'un équipement de production au sens large c'est-à-dire tout bien concourant à la production de l'entreprise De plus, le financement des PME centré sur les spéculations boursières comme alternatives possible du financement des PME à long terme c'est-à-dire supérieur à cinq ans et s'appuyant sur les immobilisations corporelles peut être une orientation de lutte contre la pauvreté en Afrique. Pour y arriver, l’objectif principal de cette étude consistera à évaluer la performance du crédit bail pour la période 1994-2008 pratiqué dans le système bancaire camerounais suite à la crise économique et financière mondiale de 2007/2008. Ce qui ne sera possible qu'en n'insistant sur le test de Durban Watson pour la période 1994-2008 évaluant ainsi, le rôle du crédit-bail et la bourse des valeurs dans le financement des PME. L’intérêt de cette étude est d’identifier des stratégies à même d'améliorer le rendement et l'efficacité du crédit-bail. Par ailleurs, les résultats des investigations contribueront à diminuer la part des demandes de crédits refusées par les banques en courte période et en longue période, les bourses prendront le relais. L'approche méthodologique insistera sur la collecte des données et l'analyse statistique et économétrique qui estime le rendement et la performance du crédit bail dans le financement du développement et la pauvreté au Cameroun. Les recommandations insisteront sur le renforcement des capacités des banques camerounaises à financer le développement et la lutte contre la pauvreté par le crédit bail.

Mots clés: Financement; crédit -bail; bourse des valeurs, micro finance; pauvreté, fiscalité

\section{Introduction}

La reprise mondiale amorcée a la suite de la crise financière de 2007/2008 s’est essoufflée, notamment dans les pays développés. Malgré les reformes mises en œuvre dans de nombreux pays développés et émergents pour résorber les effets de cette crise, le chômage a atteint des niveaux préoccupants. Les tensions sur les marchés financiers se sont accentuées essentiellement en raison des risques liés aux dettes souveraines en Europe. En outre, des signes de vulnérabilité apparaissent sur les marchés émergents. 
La hausse des prix des matières premières a porté préjudice à la croissance et a affecté les populations les plus vulnérables.

la volatilité des taux de change constitue un risque pour la croissance et pour la réduction de la pauvreté dans les pays en développement d'Afrique, en raison notamment de la persistance des déséquilibres mondiaux. Ainsi, le financement du développement se voit confronté à la raréfaction des ressources au niveau mondial. C'est pourquoi la prise de conscience d'un financement additionnel et plus assuré, est indispensable à l'atteinte des objectifs de développement survenus au niveau mondial, et conduira à la recherche des sources novatrices de financement destinées a compléter l'aide publique au développement traditionnel. C'est en ce sens qu'il convient d'être d'accord avec dial(1993), qui présente pourquoi, au Cameroun, les banques rejettent la plupart des demandes de financement d'investissements productifs émanant d'entreprises locales. A partir donc de la spécificité du marché de crédit, et les différents types de rationnement existant, Guille(1992) au même titre que Dial(1993), rappelle l'évolution du secteur bancaire camerounais depuis 1986/87 et expose les raisons qui contraignent les banquiers camerounais à refuser une part importante de la demande. En fait, les banquiers d'une part ne pouvaient pas orienter toutes les ressources dont-ils disposaient. Et d'autre part, les engagements pris étaient inférieurs à la capacité d’offre. A cet effet, constate Hugon(1996), les banquiers refusaient et refusent toujours de prêter car si le risque de défaillance se réalise, les banquiers sont dans l'incapacité de récupérer leurs créances en raison de l'inefficacité des procédures de recouvrement collectives. Ce faisant, Guille(1992) étudie les différents moyens de réduire l'asymétrie d'information: production de documents comptables et développement de la relation de clientèle pour permettre de trouver les financements complémentaires.

Au sein des pays de la zone franc, le Cameroun, constate Peyrard M. (1992), dont le PIB représente pratiquement la moitié de celui de la zone BEAC, a connu une grave crise financière en 1986/87. Ce qui a amené les banques à limiter leurs engagements. Cest en fait une inversion de tendance un peu brutale par rapport au passé où elles avaient au contraire trop prêté, et surtout sans effectuer de sélection ni de suivi des projets.

Pour éviter un effondrement de tout le système bancaire, un plan de structuration a été mis en place en 1989. Grace à ces réformes, et au changement de politique monétaire, les banques sont redevenues plus liquides, mais elles n’ont pas pour autant accordé plus de crédit à l'économie. Cette tendance n'est pas spécifique au Cameroun et concerne aussi les autres pays de la zone franc, où, bien souvent, la surliquidité des banques est encore plus prononcée. Le manque d'engagement des banques n’étant pas lié au manque de liquidité 
En vertu de la mondialisation poussée, beaucoup d'éléments ne dépendent plus seulement de l'action d'un Etat, mais d'un ensemble des Etas.

Les patrons des PME vivent l'économie et la concurrence internationale au quotidien. Ils gardent les pieds sur terre et apprécie dans qu'elle mesure le financement des PME par le crédit-bail et la bourse des valeurs influencerait le développement territorial. Ceci, car le financement des entreprises par le crédit-bail et la bourse des valeurs, est au cœur du débat sur la relance, l'emploi, la compétitivité et le développement à l'international. Les banques, partenaires privilégiées des PME, joueront le jeu du crédit dans la mesure où tout est prévisible.

En revanche, elles ne seront jamais des investisseurs audacieux de long terme ni des partenaires intéressés à la prise de risques. C'est pour ces raisons que les plus dynamiques de nos PME doivent diversifier leurs sources de financement et découvrir les vertus du marché financier dont celui de la bourse des valeurs.

La bourse, le «marché des valeurs» est un moyen de trouver rapidement de l'argent frais. C'est une opportunité qui avance lentement car le marché s'apprend, il n'est pas toujours accueillant. C'est aussi une culture.

Suivant cette perspective, en quoi peut consister le "Financement des PME" par le crédit bail ou la bourse de valeur?

Autrement dit, face à l'insuffisance des garanties de la part des demandeurs de crédit, la surliquidité des banques, en quoi peut consister le financement complémentaire ? ou en quoi peut constituer les financements alternatifs aux banques? le cas du crédit-bail (cout terme) ou bourse des valeurs (long terme). Ce qui incitera les banques à plus de crédit suivant deux moyens leur permettant de réduire leur perte en cas de défaut de l'entreprise : les fonds de garanties et la réforme des procédures collectives de recouvrement au sein de l'OHADA en train de se mettre en place dans les pays de la zone franc.

C'est au regard des questions ci-dessus, qu'il sera intéressant pour nous de dégager les objectifs ci-après :

\section{Objectifs globaux}

Le crédit-bail et la bourse des valeurs sont un outil de financement de proximité

Devant permettre de diminuer la part des demandes de crédit refusées par les banques

\section{Objectifs spécifiques}

Le crédit-bail et la bourse des valeurs sont des canaux de lutte contre la pauvreté 
Car elles permettent aux banques de réduire leur perte en cas de défaut de l'entreprise.

\section{Hypothèse}

C'est à travers le crédit-bail et la bourse des valeurs qu'on pourra vaincre la pauvreté par le financement de proximité

\section{Méthodologie}

Les données sont collectées auprès de la BEAC et du conseil national de crédit pour la période 1990-2008.Il sera question d'une analyse statistique à partir du Test Durbin Watson, afin de dégager la signification économique du financement alternatif suite au refus du crédit bancaire aux entreprises et la surliquidité des banques, conduisant à la retissance des banques à accorder des crédits et à opter le crédit-bail.

\section{Résultats attendus}

Sur le plan théorique comme sur le plan pratique, on distingue : a-Théoriquement :

Théoriquement, Peyrard M(1992) montre qu'après la crise financière de 1986/87 les banques de la zone BEAC ont limitée leurs engagements en matière d'octroi des crédits aux entreprises et sont devenues par conséquent, sur liquide et devant choisir une alternative de financement qui est sans conteste le crédit-bail.

b-Pratiquement :

Le crédit-bail contribuera à diminuer la part des demandes de crédit refusées par les banques en courte période et en longue période la bourse des valeurs prendront le relai. Ceci, en permettant aux banques de réduire leurs pertes en cas de défaut de l’entreprise qui emprunte aux banques.

\section{Plan à suivre}

On partira en partie 1, de la nécessité du crédit-bail comme outil de financement alternatif du crédit refusé par les banques en courte période et en longue période (partie2) la bourse des valeurs comme financement alternatif ou complémentaire des entreprises par les banques sur liquides.

\section{La nécessité du crédit bail dans le financement des pme Essai d'analyse.}

Selon la théorie néoclassique, dans un univers risqué, toutes les situations possibles sont connues par les agents. Ils sont omniscients et attribuent une probabilité dite objective de survenance à chaque éventualité. Le risque est équivalent à celui d'une loterie. Dans ce cadre, la banque et l'emprunteur établissent un contrat complet de type Arrow-Debreu. Ce 
contrat, appelé contingent contraignant, spécifie les actions des deux parties pour chaque état de la nature. Le problème de l'incertitude sur le comportement de l'autre est donc écarté. Les actions de chaque cocontractant sont vérifiables par un tiers, la justice.

Knight(1921), estime que les agents économiques ne connaissent pas les différents états de la nature possible, ni les probabilités d’occurrence associées. Selon son analyse, l'activité bancaire s’inscrit dans un monde incertain plutôt que dans un monde risqué. Pour faire face à l'incertitude, les banques disposent de deux instruments :

Le premier instrument consiste à prévoir la probabilité de survenance du risque à partir de l'observation de fréquences empiriques. C'est ainsi que procède les assurances.

Le deuxième instrument suggéré par Knight est de nature complètement différente. Il s'agit de l'utilisation de probabilités subjectives établies par un spécialiste. Celui-ci se distingue des autres agents par la qualité de la probabilité subjective qu’il émet. Chaque agent connait en effet ses propres compétences à établir des prédictions, mais ignore celles des autres. Il est alors tout à fait possible que deux agents qui possèdent les mêmes informations ne partagent pas les mêmes prédictions.

Le financement de l'économie à travers le crédit-bail constitue un

Moyen de financement efficace pour les PME, qui connaissent souvent des difficultés d’accès au crédit bancaire classique. Mais le financement de l’activité économique à travers le crédit bail est encore à la traîne dans la plupart des pays africains.

Pourtant, ce mécanisme d’appui à la production et à la consommation constitue l'un des produits financiers susceptibles d'améliorer le financement des PME, en ce sens qu'il permet aux entreprises de détruire des biens de production sans avoir recours au crédit bancaire.

Depuis quelques trois dernières décennies, une douzaine de pays africains, essentiellement subsahariens, rassemblés au sein d'African Leasing Association (AFROLAESE), essaient de promouvoir cette activité, pour renforcer la compétitive de leurs économies.

Sous le thème, "Crédit-bail et croissance économique », les participants au $13^{\mathrm{e}}$ congrès de cette organisation, qui s'est tenu fin Mai dernier à Yaoundé, ont réaffirmé la nécessité de promouvoir le crédit-bail, en mettant un accent sur son développement et surtout de sensibiliser et de former les promoteurs à l'utilisation de ce produit. Depuis quelques années, la banque mondiale, à travers la société financière internationale (SFI), soutient le développement du crédit bail en Afrique, d’autant que l'accès au financement pour les PME

Représente l'une des principales contraintes au développement du secteur privé en Afrique subsaharienne. Après la crise financière 
internationale, dont on connaît les conséquences sur l'économie mondiale, l'organisation des telles assises s'imposait, d'autant que « Nous considérons, à juste titre, le crédit-bail comme un outil puissant de financement des investissements donc de l'économie ", à déclaré le président de cette organisation panafricaine.

- $\quad$ Pour permettre au crédit-bail de jouer efficacement son rôle d'agent de développement en Afrique, la SFI plaide prioritairement pour l'amélioration de l'environnement fiscal et légal du crédit-bail, l’information et l'éducation des PME et du public sur le crédit-bail, la formation des acteurs et des opérateurs potentiels, le développement des nouveaux produits, un ensemble de dispositif juridique et fiscal à même d'attirer la présence de nouveaux investisseurs Le contrat proprement dit (la documentation des crédits preneurs, le nom du fournisseur, la description du bien financée, le prix du bien acquises par le crédit-bailleur au fournisseur pour être loué au crédit preneur, la durée du contrat, le nombre de matériaux à louer et leur périodicité, la valeur de l'option d'achat en fin de contrat, diverse clauses portant sur les obligations réciproques.

- $\quad$ Le tableau des échéances de loyers (y compris le cas d'échéant, une première échéance majorée faisant apparaître le montant en distinguant la TVA).

Les éventuelles garanties complémentaires ;

Etant donné que la crise financière de 1986/87 a amené les banques à limiter leurs engagements. C'est en fait une inversion de tendance un peu brutale par rapport au passé où elles avaient au contraire trop prêté, et surtout sans effectuer de sélection ni de suivi des projets. Pour éviter un effondrement de tout le système bancaire, un plan de restucturation a été mis en place en 1989. Grâce à ces reformes et au changement de politique monétaire, les banques sont redevenues plus liquide, mais elles n'ont pas pour autant accorddé plus de crédit à l'économie. Ce qu'on peut constater au tableau ci-après où statistiquement les résultats du test de Durbin Watson concernant l'évolution du crédit suivant les capitaux permanent, les besoins de financement et les dépôts obtenus au conseil national du credit.

Des préoccupations partagées par les professionnels du secteur, au regard des difficultés d'accès au financement. Aussi, proposent-ils l'élaboration d'un plan d'action pour le financement et les investissements, les changements culturels et l'amélioration du cadre juridique et fiscal. Une étude de la SFI en Europe de l'Est a démontré que, dans les marchés les plus développés, le crédit-bailleur représente environ 30\% des importations des biens d'équipement, alors que dans les économies en voie de développement, comme en Afrique, ce pourcentage est d'environ 15\%. Son apport dans le PIB à l'entreprise de couvrir ses ressources pour financer son cycle d'exploitation avec, entre autres le parement des fournisseurs et l'action des 
crédits aux clients, sans oublier qu’il est rassurant pour l'établissement de crédit, puisque ce dernier reste propriétaire du bien tout au long de la vie du contrat, ce qui lui apporte une garantie.

\section{Essai d'interprétation pratique du crédit-bail.}

Selon la Banque Mondiale(1993), le crédit-bail « repose sur l’idée que les actifs sous forme d'installations industrielles sont source de bénéfices à travers leur utilisation plutôt qu'à travers la seule propriété ».Il permet aux entreprises de détenir des biens de production sans avoir recours au crédit bancaire. Dans une opération de crédit-bail, le crédit bailleur garde la propriété du bien, ce qui lui sert de garantie immédiate en cas de défaut de l'emprunteur.

C'est également par ailleurs, l'une des formes des financements permettant à une entreprise de se doter d'un équipement de production. Il faut entendre "équipement de production » au sens large c'est-à-dire tout bien concourant à la production de l'entreprise : par exemple, du mobilier de bureau ou de l'équipement informatique, des machines industrielles, des véhicules à usage professionnel etc...

Le contrat de crédit-bail contient des documents suivants :

Tableau 1 : modèle de régression

\begin{tabular}{|c|c|c|c|c|}
\hline vannées & $\begin{array}{c}\mathrm{Y}_{\mathrm{i}}=\text { crédit à } \\
\text { l'économie }\end{array}$ & $\begin{array}{c}\mathrm{X}_{1}=\text { dépôt de } \\
\text { la clientèle }\end{array}$ & $\begin{array}{c}\mathrm{X}_{2}=\text { capitaux } \\
\text { permanents }\end{array}$ & $\begin{array}{c}\text { X3=capacité ou } \\
\text { besoin financier }\end{array}$ \\
\hline $31 / 12 / 1994$ & 6497 & 4307 & 5649 & 10956 \\
\hline $31 / 12 / 1995$ & 12102 & 6763 & 10830 & 19036 \\
\hline $31 / 12 / 1996$ & 10353 & 3055 & 11256 & 18765 \\
\hline $31 / 12 / 1997$ & 5605 & 3546 & 26735 & 18780 \\
\hline $31 / 12 / 1998$ & 9033 & 5744 & 26649 & 20223 \\
\hline $31 / 12 / 1999$ & 17126 & 5932 & 38421 & 33322 \\
\hline $31 / 12 / 2000$ & 621918 & 846504 & 81038 & 189698 \\
\hline $31 / 12 / 2001$ & 595059 & 955758 & 97400 & 230966 \\
\hline $31 / 12 / 2002$ & 661390 & 1142730 & 113195 & 385631 \\
\hline $31 / 12 / 2003$ & 717981 & 1151389 & 133099 & 340232 \\
\hline $31 / 12 / 2004$ & 828092 & 1245216 & 148169 & 438514 \\
\hline $31 / 12 / 2005$ & 908253 & 1374987 & 166454 & 491268 \\
\hline $31 / 12 / 2006$ & 915193 & 1549549 & 180610 & 672363 \\
\hline $31 / 12 / 2007$ & 963437 & 1772718 & 182310 & 837531 \\
\hline $31 / 12 / 2008$ & 1014623 & 1854448 & 169862 & 870898 \\
\hline
\end{tabular}

Source : analyse financière dynamique datée en millions de FCFA du système bancaire au Cameroun. BEAC régionale Cameroun. COBAC : secrétariat général 
Tableau 2 : Résultat de la régression :

\begin{tabular}{|c|c|c|c|}
\hline \multicolumn{4}{|c|}{ Variables introduites/éliminées (b) } \\
\hline Modèle & Variables introduites & Variables éliminées & Méthode \\
\hline 1 & CAPACITE, CAPITAUX, DEPOT (a) & & introduite \\
\hline \multicolumn{3}{|c|}{ a. Toutes variables requises introduites } \\
\hline \multicolumn{2}{|c|}{ b. variable dépendante : CREDIT } \\
\hline
\end{tabular}

Tableau 3 : Récapitulatif du modèle (b)

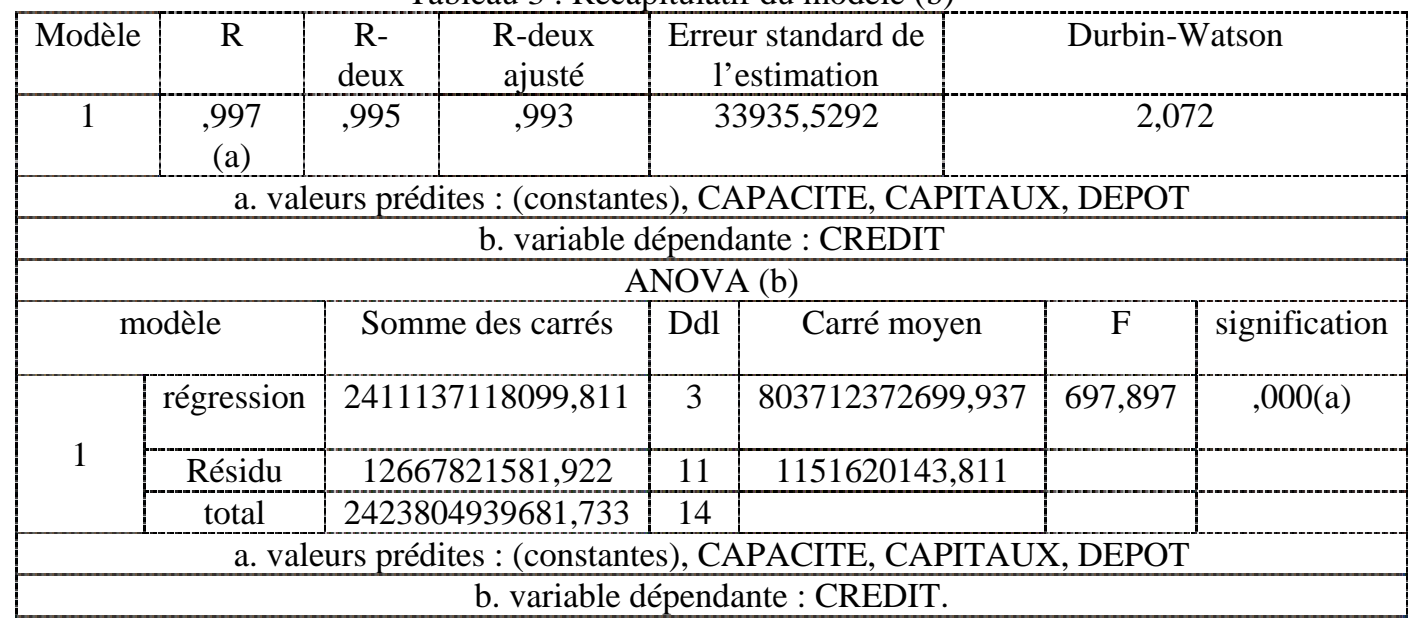

Tableau 4: Coefficients (a)

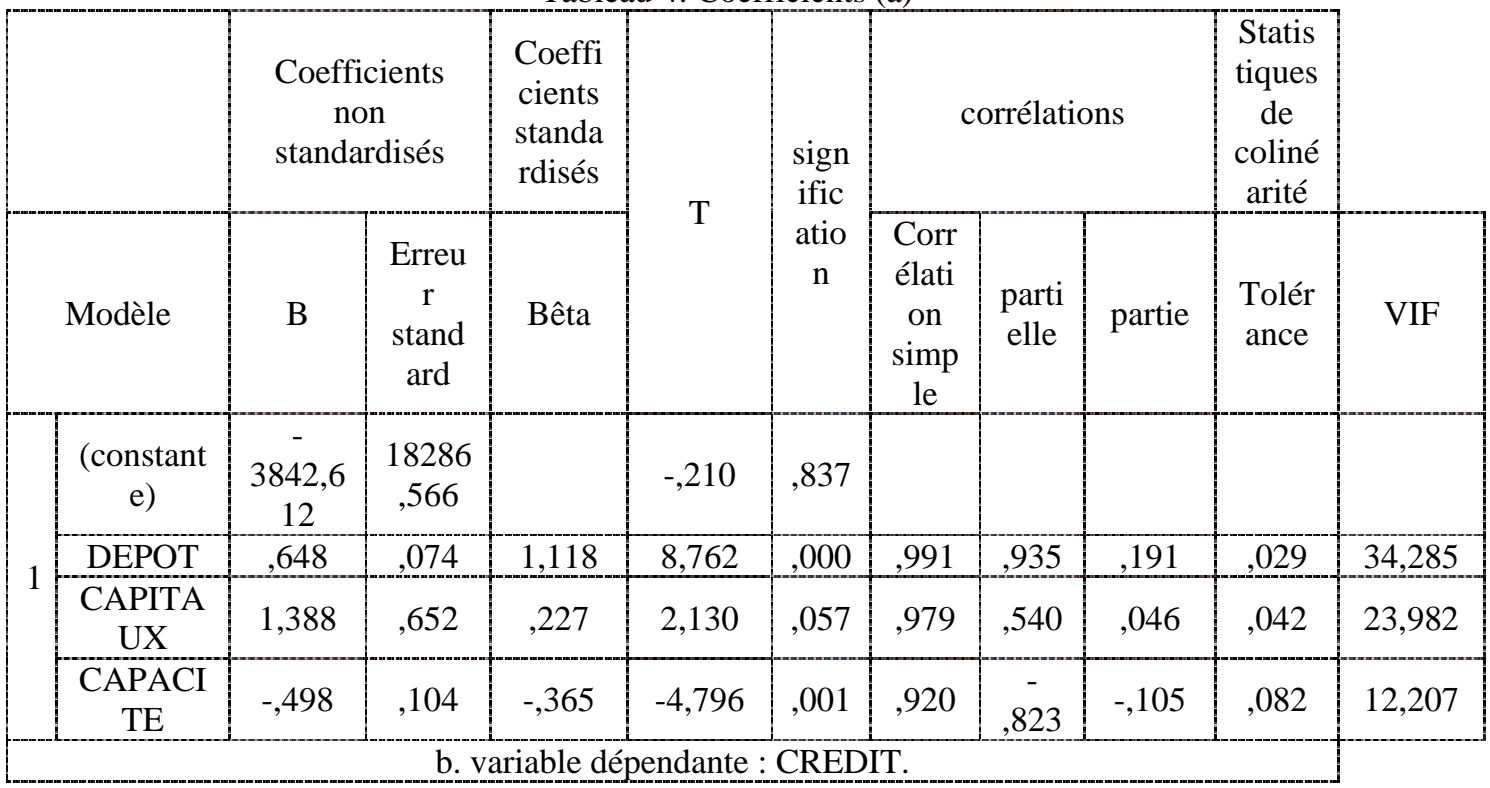

A partir d'un test de vérification (le test de Durbin Watson), on obtient un modèle économique traduisant l'évolution des crédits à la clientèle $\left(\mathrm{Y}_{\mathrm{i}}\right)$ en 
fonction des dépôts de la clientèle $\left(\mathrm{X}_{1}\right)$, des capitaux permanents $\left(\mathrm{X}_{2}\right)$, des capacités ou besoins de financement $\left(\mathrm{X}_{3}\right)$.

$\mathrm{Y}=-3.842,62+0,648 \mathrm{X}_{1}+1,388 \mathrm{X}_{2}-0,498 \mathrm{X}_{3}$

(t)' $( \pm 0,10) \quad(8,76) \quad(2,130) \quad(-4,79)$

$\mathrm{n}=15 ; \mathrm{R}^{2}=0,997 ; \mathrm{F}=697,89 ; \mathrm{D}_{\mathrm{w}}=2,072$

Avec signe.t tendant vers $0,0,000<0,05$ à $1 \% ; 0,57$ à $5 \% ; 0,001$ à $1 \%$.

$\mathrm{F}=697,89$ significatif à $1 \%$

Ces paramètres ont été calculés à partir de [95\% conf..interval]

Tableau 5 :Interprétation des paramètres

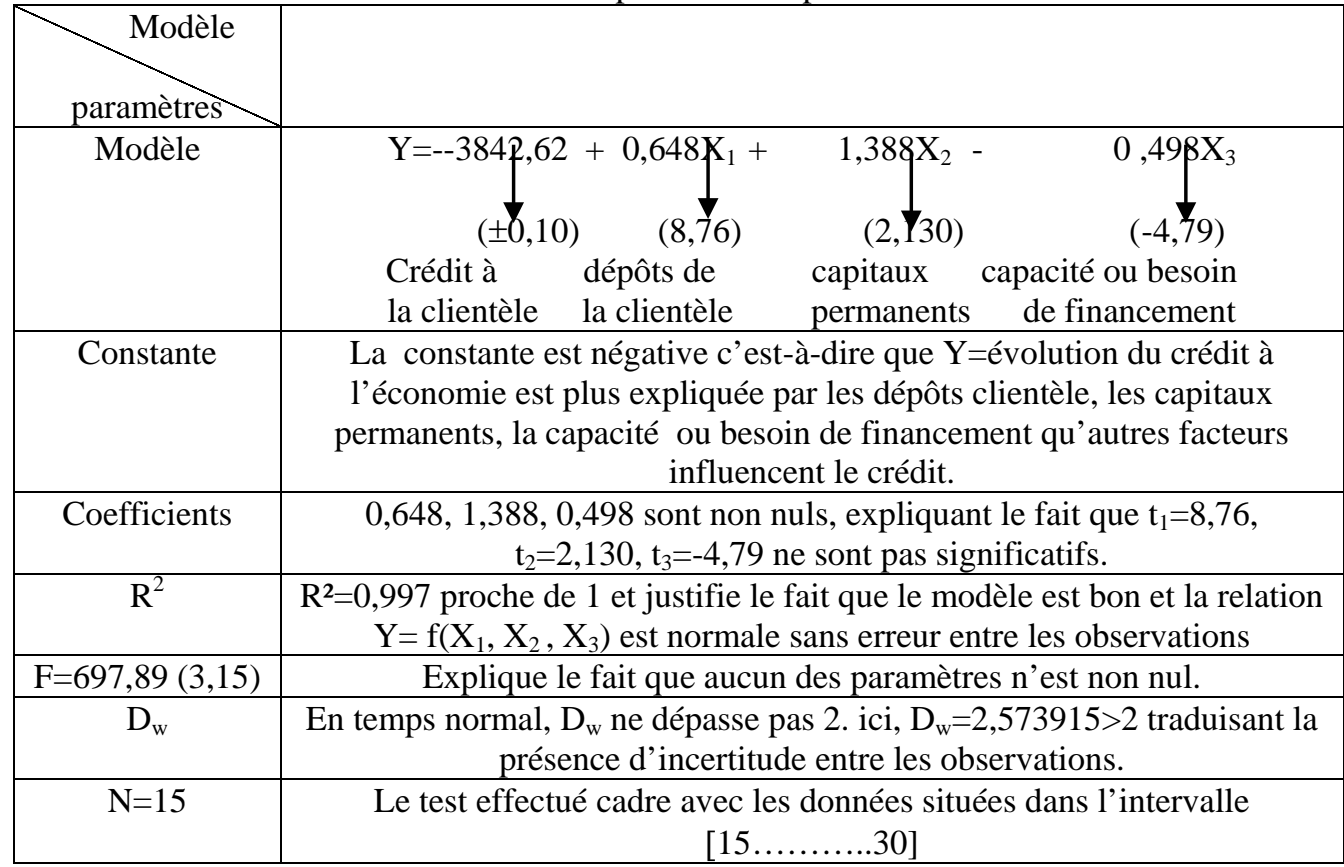

Ce test montre la nécessité d'un financement alternatif ou complémentaire au crédit bancaire face à la prudence des banques : le créditbail

\section{La nécessité de la bourse des valeurs dans le financement des pme : cas de la douala stock exchange Essai d'analyse}

La Douala stock exchange comme alternative possible du financement des PME offre une alternative de financement suivant :

- $\quad$ elle se situe dans le long terme supérieur ou égal à 5 ans et concerne les immobilisations corporelles ;

- $\quad$ ce sont les limites du financement bancaires des PME ; 
- $\quad$ la fiscalité est souple, comparée par l’accès au financement de la DSX nécessitant un coût élevé, impliquant une diversification des sources de financement ;

- $\quad$ visibilité permanente par les unités médiatiques ;

- $\quad$ obtention d'une page de transparence ;

- $\quad$ crédibilisation ;

- $\quad 28 \%$ de réductions des impôts sur les sociétés ;

- $\quad$ disponibilité de liquidité du titre ;

Ces avantages de financement des PME par la bourse de valeur disposent des contraintes du genre ;

Le cadre légal et règlementaire est exigé :

- $\quad$ Appel public à l'épargne qu'est l'action des particuliers et ceci, rien que pour les sociétés S.A. ;

- $\quad$ Recours à l'établissement de crédit et l'inscription à la bourse ;

- $\quad$ Contrôle exigé et publication des chiffres ;

- $\quad$ Appel public aux actions de la PME placé en bourse ;

- $\quad$ Transparence, information du public ;

- Vitalité des cours (variation des cours) ces contraintes supposent que la Douala Stock exchange regorge trois principaux marchés à savoir :

o Titre de capital ;

o Marché des obligations ;

o Marché hors côte.

Il faut être exclusivement une société S.A pour bénéficier des avantages de la DSX 2000 millions pour les PME donc 100 actionnaires distributions de 15\% du capital :

Le mécanisme consiste :

- $\quad$ Mettre un modèle d'accès à la bonne ;

- $\quad$ Période d'incubation deux ans ;

- Accompli les conditions d’éligibilité en bourse (20\% de capital d’entrée mais échelonné sur 10,15...ans

- $\quad$ Plan d’épargne action qui insiste sur :

o L’idée

o Les hommes

o Les moyens

Pour mettre sur pied une entreprise.

\section{Interprétation pratique}

La Douala stock exchange est l'entreprise de marché concessionnaire exclusif du service public. C’est une S.A de droit privé ayant qualité d'établissement financier. Son capital est de 1,2 millions de Francs Cfa repartie entre 
1-les prestataires de service d'investissement (55\%) (PSI) comprenant pour le moment :

- $\quad$ La BICEC ;

- $\quad$ CBC

- $\quad$ SGBC

- $\quad$ Le crédit lyonnais (Actuel SCB Cameroun)

- $\quad$ Ecobank

- $\quad$ CCEI bank (Actuel afriland First Bank)

2-Les investissements institutionnels (45\%) à la tête du marché financier camerounais ou a la commission des marchés financiers

3-(CMF), véritable gendarme qui veille sur l'épargne des camerounais ;

4-Les prestataires des services d'investissement (PSI) véritable courtier intervenant en bourse ;

La Douala Stock Exchange est le lieu de négociation des titres pour le dévouement des transactions au niveau de la banque de règlement SGBC ; pour la gestion et la conservation des titres la CAA jouant le rôle de dépositaires central.

\section{Conclusion}

Le développement du crédit-bail apparaît donc comme un soutien efficace au développement économique en Afrique Subsaharienne, en ce sens «qu'il est l'outil de financement idéal des PME ». Des Experts affirment que le crédit -bail " permet un financement à 100\% TTC »

Cela permet à l'entreprise de conserver ses ressources pour financer son cycle d'exploitation avec, entre autres, le paiement des fournisseurs et l'octroi des crédits aux clients, sans oublier qu'il est rassurant pour l'établissement de crédit, puisque ce dernier reste propriétaire du bien tout au long de la vie du contrat, ce qui lui apporte une garantie

\section{References:}

AKERLOF, G. (1984), "Labor contract as partial GB Lioiff exchange » in American theorist's book of tales. Cambridge. U. Press

ALLEMAND.S. (2005), la micro finance n'est plus une utopie! Autrement. Oxford Basic \& Black.

AMADOR- C. M.S.F.P.(2005), initiative PPTE en Afrique, Sorbonne université Paris I

ARROW K.J. (1984), Social choice and justice collected papers of K.J. ARROW, Oxford, Basic \& black well.

ARROW, K.J. (1974), Choix collectifs et préférences individuelles traduction française Paris Calman - Levy. 
AUGE, M. (1977), Pouvoir de vie, pouvoirs de mort: introduction à une Anthropologie de la répression, Paris, Flammarion.

AZZOUG, M. (1996), Recensement - diagnostic des entreprises agréées suivant la loi 1/81 : plan directeur du développement des PME,Libreville, Ministère chargé des PME,

BAD (1997), Renforcement du développement du secteur privé en Afrique, Rapport sur le développement en Afrique 1997,Abidjan, Oxford University Press.

BAD (1997), Renforcement du développement du secteur privé en Afrique, Rapport sur le développement en Afrique 1997, Abidjan, Oxford Unuveresity press.

BALLANDIER G. (1967), Anthropologie politique, Paris, PUF BANQUE MONDIALE (1984), Le miracle de l'Asie de l'Est: croissance économique et politique publique,Washington, Banque mondiale

BANQUE MONDIALE (1984), le miracle de l'Asie de l'Est : croissance économique et politique publique, Washington, Banque mondiale

BANQUE Mondiale (1996), le défi: mettre en valeur les ressources exploitées du secteur privé, République du Cameroun, 28 juin, rapport no 13955- CM, Washington, Banque mondiale.

BANQUE MONDIALE (2000) a), L'Afrique peut-elle revendiquer sa place dans le 21e siècle, Washington D.C. Banque mondiale

BANQUE MONDIALE (2000) b), Reforming publics institutions and strengthening governance, Washington.D.C. the World Bank.

BANQUE MONDIALE (2006), reforming publics institutions and strengthening governance, Washington, D.C. the world bonk.

BANQUE MONDIALE(1996), Le défi: mettre en valeur les ressources inexploitées - Evaluation du secteur privé, République du Cameroun, 28 juin, rapport $n^{\circ} 13955$ - CM. Washington D.C. Banque mondiale.

BARAT C.B. (2002), l'initiative pays pauvres très en dettes au Ghana, DIAL, mars, 39P, Washington D.C. Banque mondiale,

BARRE, R. (1956), Economie politique, Paris, PUF

BASSEY. A. (1988), African anthropology,Ibadan, Shaneson LTD

BAUDRILLARD. J. (1976), Echange symbolique et la mort. Paris, Gallimard

BAUER, P. and YAMEY, B.(1982), The effects of Aid. Encounter, March : 87 - 88, Paris, Gallimard.

BAUER, P. T.(1984). Reality and Rhetoric. Studies in the Economics of development. Paris, Gallimar

BCEAO, B. et N. (1998), « Mondialisation et intégration régionale : quelles prospectives pour le Cameroun? » in TOUNA MAMA (dir.), la mondialisation et l'économie Camerounaise, Yaoundé, édition saagraph FFE 
BCEAO. Conjoncture économique dans les pays de l’UEMOA, décembre 2001, Abidjan BCEAO.

BEAUD. M. (1993), La pensée économique depuis Keynes, historique et dictionnaire des principaux auteurs. Paris, Ed. du seuil

CIRES/RENFCAP(2014), Analyse de la performance des régies financières de la côte d'Ivoire, éd. CAPEC-CIRES-RENFCAP, Côte d'Ivoire 